\title{
LA CONEXIÓN ENTRE DERECHO Y POLÍTICA COMO JUSTIFICACIÓN DE LA RELEVANCIA DEL DERECHO. UNA APROXIMACIÓN DESDE LA PROPUESTA DE CARLOS S. NINO
}

\author{
Juan CIANCIARDO*
}

\begin{abstract}
Resumen:
Este trabajo tiene por objeto el análisis de las ideas del iusfilósofo argentino Carlos Nino acerca de la relevancia del Derecho. Concretamente se examina hasta qué punto resulta consistente el argumento según el cual su definición de Derecho como acción colectiva es efectivamente idónea para superar las llamadas paradojas de la superfluidad y de la irrelevancia del Derecho.
\end{abstract}

Palabras clave:

Derechos humanos, democracia, razonamiento jurídico, moral, paradoja de la irrelevancia.

\footnotetext{
* Investigador del Instituto Cultura y Sociedad de la Universidad de Navarra, España, y Profesor de Filosofia del Derecho en la Universidad Austral, Argentina (en uso de licencia). Agradezco las observaciones y críticas que hicieron a una versión preliminar de este trabajo los profesores Carlos Massini (Universidad de Mendoza, Argentina), Caridad Velarde (Universidad de Navarra), Pedro Rivas (Universidad de A Coruña, España), Fernando Toller (Universidad Austral, Argentina), Pilar Zambrano (Instituto Cultura y Sociedad de la Universidad de Navarra), Juan B. Etcheverry (Universidad Austral-CONICET) y Pedro Pallares (Universidad Panamericana, México).
} 


\section{JUAN CIANCIARDO}

Abstract:

The main idea of this paper is to discuss Nino's thesis about the relevance of law. Specifically, the aim is to assess Nino's definition of law -law as collective action - in light of the "paradox of the irrelevance" and the "paradox of radical indetermination" and the way it successfully -or unsuccessfully-overcomes them.

Keywords:

Human Rights, Democracy, Legal Reasoning, Moral, The Paradox of the Irrelevance. 


\section{LA CONEXIÓN ENTRE DERECHO Y POLÍTICA}

SUMARIO: I. Introducción: la doble paradoja. II. La relación directa entre el Derecho y la politica como salida de las dos paradojas. III. El Derecho como práctica colectiva y su relevancia.

\section{INTRODUCCIÓN: LA DOBLE PARADOJA}

Luego de postular la existencia de una relación justificatoria e interpretativa entre derecho y moral, el filósofo argentino Carlos Nino detectó dos "aparentes paradojas" que se seguirian de ese vínculo y que producirian (según explicó) "el riesgo de hacer irrelevante el derecho en el razonamiento práctico". ${ }^{1}$ Al análisis de uno y otro problema se dedicará este trabajo. Nino los trató en dos oportunidades, con argumentos no coincidentes - aunque no contradictorios-. A1 primero de esos trabajos dediqué un estudio reciente. ${ }^{2}$ En esta oportunidad, por eso, me concentraré en el segundo. ${ }^{3}$

1 Nino, Carlos, Derecho, Moral y Politica. Una revisión de la teoría general del Derecho, Barcelona, Ariel, 1994, p. 130.

2 Cfr. Cianciardo, Juan, "La paradoja de la irrelevancia moral del gobierno y del Derecho. Una aproximación desde el pensamiento de Carlos Nino", Díkaion. Revista de fundamentación jurídica, Colombia, año 24, vol. 19, núm 1, junio de 2010, pp. 47-70. El primer acercamiento, en Carlos Nino, "La paradoja de la irrelevancia moral del gobierno y el valor epistemológico de la democracia”, Análisis Filosófico VI-1, 1986. Este trabajo fue publicado luego en Nino, Carlos, El constructivismo ético, Madrid, Centro de Estudios Constitucionales, 1989, pp. 111-133. Existe, asimismo, una versión en inglés con algunas diferencias respecto del texto inicial: “The Epistemological Moral Relevance of Democracy", Ratio Iuris, vol. 4, núm. 1, 1991, pp. 36-51.

3 Con respecto a la expresión "la paradoja de la irrelevancia", cfr. Ródenas, Ángeles "Sobre la justificación de la democracia en la obra de Carlos S. Nino”, Doxa, núm. 10, 1991, pp. 279-293; de la misma autora, Sobre la justificación de la autoridad, Madrid, CEC, 1996, pp. 226 y ss. Asimismo, Greppi, Andrea, "Consenso e imparcialidad. Sobre la justificación moral de la democracia en el pensamiento de C. S. Nino", en Rosenkratz, Carlos y Vigo, Rodolfo (comps.), Razonamiento jurídico, ciencia del derecho y democracia en Carlos S. Nino, México, Fontamara, 2008, pp. 


\section{JUAN CIANCIARDO}

La primera paradoja es la de la superfluidad del Derecho. Nino la explica con estas palabras: "si para que una norma jurídica justifique una acción o decisión debemos inevitablemente mostrar que emana de principios morales que se asumen como válidos ¿por qué no buscar la justificación de esa acción o decisión directamente en esos mismos principios? ¿Para qué necesitamos, al fin y al cabo, un gobierno y sus leyes si éstas no permiten justificar una acción más que recurriendo, en última instancia, a principios morales?". ${ }^{4}$ Efectivamente, "si las leyes coinciden con tales principios son redundantes, y si no coinciden no se les debe prestar atención". 5

Existen dos respuestas para este primer problema que, según Nino, no son satisfactorias. Se podría sostener, indica, que el Derecho resuelve los casos de indeterminación moral, y que alli radica su relevancia. "Sin embargo, no es fácil vislumbrar cómo las lagunas de los principios morales puedan llenarse sin recurrir a otros principios morales". ${ }^{6}$ Es decir, para resolver las indeterminaciones morales no puede prescindirse de consideraciones morales, que el Derecho no podría aportar. Por ende, el argumento expuesto no permitiría superar esta formulación de la paradoja de la irrelevancia. Por otro lado, según una segunda respuesta posible, la justificación de las leyes "generalmente no incluye consideraciones morales sustantivas sino que es una justificación de tipo procesal", 7 esto es, una justificación que se limita "a determinar las condiciones para la selec-

221-259, esp. pp. 232-238. Raz alude a esto mismo en Raz, Joseph, Razón práctica y normas, trad. de J. Ruiz Manero, Madrid, Centro de Estudios Constitucionales, 1991, pp. 242, 155 y passim; más recientemente, cfr. Raz, Joseph, "About Morality and the Nature of Law", American Journal of Jurisprudence, Estados Unidos de América, vol. 48, 2003, p. 1, y, en alguna medida, "Respect, Authority \& Neutrality", Oxford Legal Studies Research Paper, núm. 30, 2010.

4 Nino, Carlos, Derecho, Moral y Politica (...), op. cit., pp. 130-131.

5 Idem.

6 Ibidem, p. 132.

7 Ibidem, pp. 131-132. 
ción de las autoridades politicas y del procedimiento bajo el cual éstas deben actuar, de modo que cuando son satisfechas, las leyes resultantes están justificadas". 8 Siendo ello así, la relevancia del Derecho residiría en su capacidad para resolver los conflictos, una vez traspuesto ese umbral (bajo, minimo) de condiciones. Sin embargo, sostiene Nino, no "resulta evidente cómo detectar principios morales que toman como justificación última la adopción de ciertos procedimientos. Por ejemplo, la mayoría de las justificaciones de la democracia sólo son procesales en una primera instancia, pero luego proceden a justificar los procedimientos relevantes a la luz de cierto bien sustantivo como la autonomía o la utilidad, la materialización del cual podría, en principio, darse con independencia del procedimiento en cuestión". ${ }^{9}$ En suma, las dos respuestas más habituales o inmediatas a la paradoja de la superfluidad no superan la objeción.

La segunda paradoja consiste, también, en un cuestionamiento de la relevancia del gobierno y de sus leyes. Se trata de una paradoja causada por la "indeterminación radical" del Derecho. Nino explica que, si se abstraen todos los pasos valorativos de la interpretación, el único "dato duro" que podría condicionar el proceso interpretativo consiste en textos o conductas, es decir, en gráficos o movimientos corporales. "Dadas las opciones de criterios generales de interpretación para dotar de significado a tales entidades o eventos, las alternativas para superar las imprecisiones o ambigüedades de esos significados, las variantes para superar las indeterminaciones lógicas, los textos y conductas en cuestión pueden estar asociadas con cualquier contenido proposicional, según los principios valorativos asumidos en cada paso". ${ }^{10}$ Sin acudir a consideraciones extra-normativas ("extrajurídicas" las llama Nino) no parece posible superar la opacidad de los textos. Esto conduce a nuestro au-

8 Ibidem, p. 132.

9 Idem.

10 Ibidem, p. 133. 


\section{JUAN CIANCIARDO}

tor a preguntarse, nuevamente, por la relevancia del derecho positivo, "entendido como actos lingüísticos, textos o prácticas, dado que las proposiciones que les asignamos como significados van a depender totalmente de los principios valorativos que adoptemos. ¿Por qué no acudir directamente a tales principios valorativos para obtener las soluciones deseadas, en lugar de hacerlas aparecer como si surgieran del texto?". ${ }^{11} \mathrm{El}$ intérprete nada podría hacer con el Derecho sin que su interpretación se nutra de la Moral. Incluso, lo realmente significativo de su tarea provendría en última instancia de alli.

En suma, si el Derecho tiene una relación justificatoria con la Moral, entonces es superfluo; y si tiene una relación interpretativa, entonces es irrelevante. La primera de las paradojas surge en el plano de la normatividad del Derecho, la segunda, en el de su interpretación. Se trata, en realidad, de dos formas de aproximarse al mismo problema, de dos modos de cuestionar la aportación del Derecho al razonamiento práctico. Nino afirma la existencia de la doble relación mencionada (justificatoria e interpretativa) y debe, por tanto, resolver una y otra dificultad. Debe, en definitiva, intentar fundamentar cuál es la diferencia práctica que el Derecho aporta a la Moral. En su segunda aproximación al problema, a la que, como anticipé al comenzar, se dedicarán las próximas páginas, el autor argentino sostiene que la solución estriba en la aceptación de la existencia de una relación directa entre el Derecho y la Política.

11 Ibidem, pp. 133-134. Nino desarrolló en otro trabajo suyo una crítica profunda y sugerente de lo que definió como una "preocupación recurrente de los juristas dogmáticos acerca del carácter científico de su actividad", preocupación que explicaría actitudes como la que implícitamente se critica con la pregunta formulada en el texto. Luego de negar la pretendida cientificidad (more moderno) para el Derecho, Nino propuso el reemplazo de dicha preocupación por "una búsqueda legítima de analogías y diferencias con las actividades reconocidas como cientificas". Cfr. Nino, S. Carlos, Consideraciones sobre la dogmática jurídica (con referencia particular a la dogmática penal), México, UNAM, 1989, passim. 


\section{LA RELACIÓN DIRECTA ENTRE EL DERECHO Y LA POLÍTICA COMO SALIDA DE LAS DOS PARADOJAS}

Según Nino, las dos paradojas descriptas precedentemente desaparecen si se concibe al Derecho como una práctica colectiva (concretamente, como una práctica política). Veremos a continuación en qué consiste esta concepción, para pasar luego al examen de su viabilidad como explicación de la relevancia del Derecho.

\section{El Derecho como práctica colectiva}

En opinión de Nino, el Derecho sólo se comprende bien como acción colectiva. Únicamente desde allí puede explicarse su relevancia. Para desarrollar esta idea propone varias analogías. Se vale, sobre todo, de la comparación entre el trabajo del operador jurídico y la participación de un músico en un conjunto o una orquesta. Nino sostiene que un buen músico posee, obviamente, sus propios criterios acerca de cómo debe interpretarse un cuarteto o una sinfonía. "Sobre la base de esa valoración, el músico juzgará los méritos de la ejecución conjunta y, si su valoración difiere de la de los demás integrantes del conjunto, tratará de convencerlos para que ajusten la contribución de cada uno de ellos a la obra colectiva a los criterios que él defiende". ${ }^{12} \mathrm{Si}$ el músico no convenciera a sus compañeros, podrían ocurrir dos cosas: que decidiese no participar de la obra, o que, por el contrario, decidiese participar, sobre la base de que la ejecución tiene algún valor, aunque no coincida completamente con su gusto. Nino continúa diciendo que "al decidir esto, sería absurdo que el músico de nuestro ejemplo realizara su contribución según sus particulares valoraciones si ello produjera una falta de sintonía con la contribución de los otros músicos". ${ }^{13}$ Dicho con otras palabras, si al

12 Ibidem, p. 135.

13 Idem. 
músico le interesara que su contribución fuese eficaz, debería ajustarla a las concepciones estéticas de los demás. ${ }^{14}$ Por eso, sostiene Nino, "es necesario que tenga en cuenta el estilo que los otros músicos imprimen a su ejecución y que seguirán imprimiendo en el transcurso de la misma. Es posible que la obra le dé algún margen que le permita expresar, en su contribución, su propia concepción estética y aproximar así la ejecución conjunta a su ideal musical. Sin embargo, la expresión de su propia concepción se verá limitada por la necesidad de una ejecución armoniosa, en la medida en que juzgue que esa ejecución tiene algún valor, y que no tiene posibilidades reales de participar de una ejecución colectiva que se aproxime aún más a su propia concepción estética". 15

Del ejemplo surge, sigue diciendo Nino, "que hay una racionalidad especifica al participar en obras colectivas. Cualesquiera que sean los criterios o modelos que definen lo que es óptimo en la clase de cosas o fenómenos a los que pertenece la obra colectiva, esos modelos o criterios resultan modificados cuando se aplican a una acción que contribuye a una obra sin tener el control absoluto sobre la misma". ${ }^{16}$ En este tipo o clase de acciones surge un nuevo modo de racionalidad, "puesto que, en tanto nuestra aportación a una obra colectiva es limitada y no controlamos el producto final, lo racional puede ser elegir, no el modelo o principio más defendible, sino otros con méritos menores. Este tipo de racionalidad, constreñida en la obra colectiva por las elecciones de los otros, podría ser denominada de "lo segundo mejor", puesto que, muchas veces, nos lleva a un alejamiento progresivo del modelo óptimo, en atención a sus efectos sobre la obra global". ${ }^{17}$

Partiendo de este ejemplo y de esta conclusión, nuestro autor propone concebir la tarea del operador jurídico como

14 Idem.

15 Idem.

16 Ibidem, p. 136.

17 Ibidem, p. 137. 
una participación en una obra colectiva. ${ }^{18} \mathrm{El}$ objeto de esa obra colectiva - de la que participarian constituyentes, legisladores, jueces y administradores- "es el desarrollo del derecho vigente como parte de un proceso todavía más amplio, como lo es el complejo de prácticas, instituciones, costumbres, actitudes culturales y creencias básicas que definen a una sociedad". 19

\section{Un razonamiento juridico en dos niveles}

Nino propone, entonces, reconocer "el hecho de que la acción de los legisladores y jueces se desarrolla en el contexto de una obra colectiva cuyos restantes aportes ellos no con-

18 La conexión entre esta idea y la conocida imagen de la "novela en cadena" que propuso el Profesor R. Dworkin es evidente. Aunque el estudio de este punto desborda el objeto que aquí me he propuesto, conviene dejar constancia de lo que Nino señala al respecto. Según él mismo dice, su enfoque difiere del de Dworkin y de los de otros autores en que "sólo parte del reconocimiento del hecho de que la acción de los legisladores y jueces se desarrolla en el contexto de una obra colectiva cuyos restantes aportes ellos no controlan. Este enfoque es perfectamente compatible con la conclusión de un juez o de un legislador acerca de que el derecho vigente hasta ese momento es tan precario, que vale la pena el esfuerzo de arriesgar una refundación, e incluso justifica, si ese esfuerzo fracasa o es imposible, una situación de anarquía o de disgregación del orden jurídico. Lo que simplemente toma en cuenta es la circunstancia de que, si la anterior no es una alternativa aceptable, la decisión del juez debe concebirse como parte integral de un orden jurídico, fundado en un cierto hecho constitucional" (ibidem, pp. 138-139). La propuesta del Profesor Dworkin, en A Matter of Principle, Clarendon Press, Oxford, 1986, pp. 146-166 y Law's Empire, Cambridge Mass., Harvard University Press, 1986, pp. 228-238. En el primero de estos trabajos Dworkin dice, expresamente, que su concepción del sistema jurídico como un ejercicio de interpretación es "profunda y completamente politica", p. 146. La bibliografia existente sobre esta propuesta es abrumadora. Puede $c f r$., al respecto, Zambrano, Pilar, "Objetividad en la interpretación judicial y objetividad en la moral. Una reflexión a partir de las luces y sombras en la propuesta de Ronald Dworkin”, Persona y Derecho, España, vol. 56, 2007, pp. 281-326.

19 Nino, Carlos, Derecho, Moral y Politica (...), op. cit., p.137. 
trolan". ${ }^{20}$ Este hecho es independiente de que un legislador o un juez consideren que el Derecho existente es tan deficiente que vale la pena refundarlo o incluso desobedecerlo. "Lo que simplemente toma en cuenta es la circunstancia de que, si la anterior no es una alternativa aceptable, la decisión del juez debe concebirse como parte integral de un orden jurídico, fundado en un cierto hecho constitucional". ${ }^{21}$ Dicho de otro modo, lo que debe reconocerse es que "si la única forma de tomar decisiones moralmente justificadas ha de ser en el contexto de prácticas sociales existentes, se hace necesario preservar el orden jurídico vigente, salvo que sea tan injusto que no pueda ser mejorado y que la única decisión moralmente justificada es la que conduce a desconocerlo, aun a riesgo de no poder establecer otro con éxito". ${ }^{22} \mathrm{El}$ autor sostiene, a partir de las ideas precedentes, que el enfoque propuesto por él "revela que el razonamiento jurídico muestra una estructura escalonada en dos niveles": 23

a) un nivel primario y básico, en el que "se deben articular las razones que legitiman o no la práctica social constituida por el derecho positivo". ${ }^{24}$ Rigen aquí "razones sustanciales y procedimentales que permiten ubicar a ese derecho en un continuo de legitimidad". 25

Volviendo a la analogía empleada párrafos más arriba, en este nivel se trataría de establecer si los criterios estéticos de la orquesta en su conjunto son en términos generales conciliables con los que tiene personalmente el músico, más allá de que puedan no coincidir por completo.

b) Una vez superado el primer nivel del razonamiento jurídico (es decir, una vez que se ha concluido que el orden jurídico es legítimo, incluso aunque pueda ser imperfecto),

20 Ibidem, p. 138.

21 Ibidem, p. 139.

Ibidem, p. 140.

Idem.

24 Idem.

25 Idem. 
se pasa al segundo nivel en el que "se trata de aplicar ese orden jurídico para justificar acciones o decisiones". ${ }^{26}$ Este segundo nivel se desarrolla en el contexto proporcionado por el primero: "quedan excluidas las razones justificatorias (...) incompatibles con la preservación del derecho positivo (...) si es que aquel razonamiento ha arrojado como resultado que ella es más legítima que cualquier alternativa realista". ${ }^{27}$ Por esa razón, "un principio que tiene impecables credenciales desde el punto de vista de los criterios de validación implícitos en nuestro discurso moral puede ser, sin embargo, descalificado o exceptuado si ello resulta necesario para preservar la vigencia del sistema jurídico". ${ }^{28}$ Retornando nuevamente a la analogía, si el músico ha encontrado en el primer nivel de razonamiento razones suficientes para tocar en esa orquesta concreta, es posible que en el segundo nivel deba dejar de lado - al menos en alguna medida - su criterio estético personal en la interpretación concreta de la pieza que le toca.

Los principios morales influyen en los dos niveles. En el primer nivel actúan como "último tribunal de apelación". En el segundo adquieren una relevancia decisiva "en la medida en que no sean incompatibles con las conclusiones alcanzadas en el primer nivel". ${ }^{29}$ De lo contrario, señala Nino, "serian autofrustrantes, puesto que neutralizarian las conclusiones alcanzadas mediante su propio empleo". ${ }^{30}$

Abordando el mismo tema desde un ángulo distinto, podría interpretarse que Nino se plantea dos preguntas fundamentales: a) ¿para qué el Derecho en general?; b) ¿por qué y para qué esta norma en relación con esta conducta o decisión en particular? La respuesta a la primera pregunta, desde esta perspectiva, condiciona la respuesta a la segunda. Se trata, en mi opinión, y más allá de las críticas que se

26 Ibidem, p. 141.

27 Idem.

28 Idem.

29 Idem.

30 Idem. 
harán al final, de un planteamiento en general acertado, que parte de la consideración del Derecho como praxis, y sobre esa base confiere primacía a su fin. ${ }^{31}$ La descripción de un producto humano - y el Derecho lo es- no permite su explicación. Por ejemplo, la descripción de un reloj (¿en qué consiste?): una esfera, dos agujas que giran a distinta velocidad, etc., no permite su explicación (¿qué es un reloj?). Para poder dar cuenta de esto último, es necesario preguntarse para qué fue producido. Del mismo modo, la sola descripción del fenómeno jurídico no permite, tampoco, explicarlo ni, mucho menos, comprenderlo. ${ }^{32}$ La comprensión sólo puede alcanzarse sobre la base de la consideración de los fines últimos del Derecho como práctica. Esto y la conexión entre descripción, explicación y comprensión explica que, como afirma Dworkin, una respuesta acerca de cuáles son esos fines se encuentre al menos de modo implícito en todos aquellos que participan del Derecho. ${ }^{33}$

Sobre la base proporcionada por estas ideas, Nino se propone, entonces, refutar las dos paradojas mencionadas al comienzo.

31 De Lucas, Javier, "La pregunta fundamental una precisión metodológica”, Persona y Derecho, España, vol. 9, 1982, pp. 121-125.

32 Cfr., al respecto, Cotta, Sergio, El Derecho en la existencia humana. Principios de ontofenomenología jurídica, Pamplona, Eunsa, 1987, pp. 21-28. [ed. española a cargo de I. Peidro Pastor].Sobre este autor, cfr. Persona y Derecho, España, vol. 57, 2007, dedicado monográficamente a su figura y a su pensamiento. Señala Cotta: "cuando algo se produce, la primera indagación que cabe es la del fin que persigue el sujeto que lo produce. Tal es el fundamento de la llamada interpretación teleológica o finalista, capaz de aclararnos el hecho producido atendiendo al fin que se ha deseado (...). Sin duda, el Derecho (...) es un producto cultural humano, en la significación más simple y usual de producto: son los hombres quienes producen las leyes, sentencias, contratos, etc. Y los producen para servir una determinada finalidad abstracta y para aplicar a innumerables casos concretos" (op. cit., pp. 21-22).

33 Cfr. Dworkin, Ronald, Law's Empire, op. cit., pp. 31-44 y 65-68, entre otros lugares. 


\section{La superación de la paradoja de la irrelevancia en el razonamiento práctico}

Sostiene el autor argentino que "la concepción del derecho positivo como una acción colectiva extendida en el tiempo a la que deben aplicarse los principios de justicia y de moralidad social, en lugar de aplicar directamente esos mismos principios a cada contribución a la práctica, permite (...) superar la paradoja de la irrelevancia del derecho positivo en el razonamiento práctico". ${ }^{34}$

En efecto, "la referencia al derecho positivo resulta ineludible en el razonamiento práctico dirigido a justificar acciones y decisiones, ya que sin esa referencia la aplicación de los principios morales y de justicia tiene un objeto inapropiado. Los principios en cuestión tienen, como materia de valoración, prácticas sociales como el derecho positivo, y no la acción de un individuo aislado. Por lo tanto, el derecho positivo, aunque no provee razones últimas para justificar acciones o decisiones - que sólo están provistas de aquellos principios aceptados autónomamente-, constituye el objeto de referencia de tales razones y una etapa intermedia obligatoria en el razonamiento práctico que conduce a una decisión". ${ }^{35}$

Retornando a la analogía, en opinión de Nino el objeto de la práctica social en la que consiste el Derecho no es el ideal estético del músico del ejemplo - la moral o los principios morales-, sino los criterios estéticos con los que toca la banda - el derecho positivo-. De alli que el profesor argentino sostenga que obviar el Derecho positivo supondría un error de objeto. El acierto de una decisión que se adopta en el contexto de una práctica colectiva no depende exclusivamente de la opinión de quien decide, sino también de los criterios y principios que surgen de ese contexto. Utilizando un conocido ejemplo de Dworkin: el intérprete que se pregunta acerca de si está ordenado o prohibido en una comu-

34 Nino, Carlos, Derecho, Moral y Politica (...), op. cit., p. 145.

35 Idem. 
nidad determinada ingresar a la iglesia con sombrero no puede prescindir en su respuesta de la praxis seguida hasta ahora por los miembros de esa comunidad. ${ }^{36}$ Esa praxis constituye, en realidad, el objeto concreto de su indagación. No lo es de modo inmediato, en cambio, su opinión personal acerca de la justicia o conveniencia de una norma de esa naturaleza. Dicho con otras palabras, según Nino, cuando el intérprete se pregunta ¿cómo debo resolver este caso?, en la misma pregunta está la referencia al Derecho positivo, pues es imposible ser justo sin atender al criterio de justicia que la sociedad ha acogido a través del él.

\section{La superación de la paradoja de la indeterminación} radical del derecho positivo

Esta paradoja es el resultado de la percepción del papel central que ocupan las valoraciones en el proceso de interpretación jurídica.

En opinión de Nino "una vez que reconocemos que el razonamiento jurídico presenta esta estructura escalonada, que la racionalidad correspondiente es del tipo de la segunda mejor posición y que los principios morales y de justicia no tienen por objeto acciones y decisiones individuales sino prácticas o acciones colectivas, entonces el derecho ya no aparece como una caja negra asimilable, mediante cualquier solución, por nuestras valoraciones, sean las que sean, sino como una práctica que incluye criterios convencionales de interpretación de textos y otros materiales jurídicos relevantes. Esto implica mostrar que la paradoja de la indeterminación radical del Derecho sólo se da cuando concebimos los actos y decisiones jurídicos como si fueran actos y decisiones aislados". ${ }^{37}$

36 El ejemplo, aunque aplicado a otro problema, en Dworkin, Ronald, Taking Rights Seriously, Duckworth, Londres, 1977, cáp. 2.

37 Nino, Carlos, Derecho, Moral y Politica (...), op. cit., p.147. 
Retrocedamos un momento en la argumentación para comprender mejor lo que propone Nino. La paradoja a resolver podría sintetizarse así: si la interpretación jurídica es radicalmente indeterminada, y su determinación sólo puede hacerse recurriendo a la Moral, ¿por qué el Derecho? ¿Por qué no, más bien, sólo la Moral? Aunque Nino no lo dice con estas palabras, lo cierto es que su propuesta consiste, en última instancia, en afirmar que la indeterminación jurídica no es "radical". Es decir, que no cualquier interpretación jurídica es aceptable, y que la aceptabilidad de una interpretación depende del Derecho positivo y no de la Moral, puesto que no se trata de una acción individual llevada adelante por un intérprete concreto, sino una práctica colectiva.

\section{EL DERECHO COMO PRÁCTICA COLECTIVA Y SU RELEVANCIA}

\section{Volviendo a la introducción: el interés del tema}

Llegados a este punto corresponde llevar a cabo algunas observaciones acerca del problema que hemos analizado y la respuesta ofrecida por Nino. Lo primero que vale la pena afirmar es su interés. No se trata de una cuestión puramente teórica, o de un pseudo-problema analítico, como parecería sugerir una aproximación superficial.

El eje del debate iusfilosófico de los últimos cincuenta años ha sido la tesis de la conexión entre Derecho y Moral, que osciló y oscila aún entre su aceptación o su rechazo. Entre quienes aceptan la conexión se da una gran gama de matices. La aceptación de la tesis de la conexión conduce a la pregunta por la relevancia del Derecho. Es decir, si hay conexión, cabe preguntarse entonces cuál es la relevancia del Derecho, porqué el Derecho y no únicamente la Moral. Es obvio que la aceptación implica - al menos implícitamente- diferenciación. Si no hubiera diferenciación no habría conexión sino confusión o identificación. Si hay conexión, es porque hay diferenciación. Y si hay diferenciación, 
esa diferenciación debe ser relevante, puesto que de lo contrario uno de los dos términos perdería total o parcialmente su sentido -y tampoco podría hablarse plenamente de conexión-.

La pregunta, en síntesis, es interesante, aunque la respuesta de Nino contenga algunas inconsistencias.

\section{Una gama amplia de respuestas problemáticas}

Veamos muy sintéticamente las posiciones que niegan directa o indirectamente la relevancia. Existen al menos tres alternativas.

Una primera posibilidad es negar la relevancia del Derecho sobre la base de que la Politica es capaz de proveer autónomamente de razones para actuar. Es la postura, por ejemplo, de algunos autores del Critical Legal Studies Movement. Para Duncan Kennedy, por ejemplo, "la decisión judicial funciona para asegurar ya intereses ideológicos particulares, ya intereses generales de clase de la intelligentzia, relacionados con el statu quo social y económico". ${ }^{38}$ Desde esta perspectiva, los juristas deberian practicar, "una hermenéutica de la sospecha en relación con la afirmación de que el discurso jurídico, y particularmente los argumentos jurídicos, (...) son autónomos respecto del discurso ideológico". ${ }^{39}$ El Derecho, en definitiva, carece de relevancia: lo verdaderamente importante es el discurso ideológico que aparece oculto tras su fachada.

Una segunda opción consiste en relativizar hasta opacar la relevancia del Derecho sobre la base de que sólo la Moral es capaz de proveer razones excluyentes para actuar. Esta

38 Kennedy, Duncan, Izquierda y derecho. Ensayos de teoría jurídica critica, trad. Guillermo Moro, Buenos Aires, Siglo Veintiuno, 2010, p. 29. Para una consideración global de lo que ha aportado este autor a la corriente en la que se enrola, cfr. Funakoshi, Motoaki, "Taking Duncan Kennedy Seriously: Ironical Liberal Legalism”, Widener Law Review, USA, vol. 15, núm. 1, 2009, pp. 231-287.

39 Kennedy, Duncan, Izquierda y derecho (...), op. cit., p. 30. 


\section{LA CONEXIÓN ENTRE DERECHO Y POLÍTICA}

posición ha sido sostenida, históricamente, por el iusnaturalismo racionalista o por algunas de sus derivaciones. ${ }^{40}$

En ambas alternativas el resultado final — si se siguen coherentemente todas las consecuencias- es el nihilismo jurídico, postulando el reemplazo del Derecho por un gobierno que en la práctica no tiene límites o por la anarquía, respectivamente. Si el Derecho es en realidad poder, no hay nada que limite el poder. Si el Derecho es en realidad Moral, entonces el Derecho y, a fortiori, el gobierno carecen de justificación.

Existe otro conjunto de autores que sin llegar a negar por completo la relevancia del Derecho la reducen tanto que acaban privando de sentido a las normas de alcance general, y postulan de manera directa o aceptan de hecho el gobierno de los jueces. ${ }^{41}$ Este fenómeno, muy presente en al-

40 La falta de distinción entre Derecho y Moral se observa, por ejemplo, en Cathrein, Victor, El Derecho natural y el positivo, 2a. ed., trad. de A. Jardón y C. Barja, Madrid, Reus, 1926, pp. 268-286, y passim. Sostiene este autor que "el Derecho es una parte esencial del orden moral" (272), y que "no se puede abarcar enteramente el orden moral sin referirlo al orden del Derecho" ni, inversamente, "comprender el orden de Derecho sin entrar inmediatamente en el campo de lo moral", p. 274. Afirma, asimismo, que "como el restante orden moral, también el orden jurídico tiene su raíz y fundamento en la voluntad racional de Derecho, ordenadora del mundo; el cumplimiento de los designios divinos es su fin, y en la voluntad divina tiene su limite infranqueable", p. 277. Cfr. sobre este autor, recientemente, Brieskorn, Norbert, "Victor Cathrein S. J. Naturrechtliche Strömungen in der Rechtsphilosophie der Gegenwart", Archivfürrechtsundsozialphilosophie, Alemania, núm. 116, 2009, pp. 167-185.

41 Este fenómeno se ha potenciado especialmente en el Neoconstitucionalismo, de la mano del auge del control de constitucionalidad, que genera una "tensión", según Roberto Gargarella, entre "la organización democrática de la sociedad, y la función social de revisión de las leyes". Cfr. sobre este tema, en general, Gargarella, Roberto, La justicia frente al gobierno. Sobre el carácter contramayoritario del poder judicial, Barcelona, Ariel, 1996 (la cita en p. 13) y, más recientemente, "De la alquimia interpretativa al maltrato constitucional. La interpretación del Derecho en manos de la Corte Suprema argentina”, en Espinoza de los Monteros, Javier y Narváez Hernández, José Ramón, Interpretación jurídica: modelos históricos y realidades, México, UNAM, 2011. 


\section{JUAN CIANCIARDO}

gunos países de Iberoamérica, suele ir de la mano de una crisis profunda de la representación política. El juzgado se transforma en una oficina de reclamos en la que se atienden todos aquellos asuntos que los restantes órganos de gobierno no resuelven pese a que no los elige la población, ni que el proceso que da lugar a la elaboración de la sentencia tenga puntos de contactos relevantes con el debate democrático.

Frente a las dificultades que presentan estas diferentes alternativas, Nino cree ver una propuesta superadora en su comprensión del Derecho como acción colectiva. Su atractivo radicaría en lo siguiente: frente al pluralismo de concepciones morales, el Derecho permitiría que cada agente moral actuara de acuerdo con el conjunto de la sociedad, impidiendo, precisamente, la anarquía social a la que conduciría esa anarquía moral de base.

\section{La posición de Nino y el debate entre excluyentes} $e$ incluyentes, $y$ entre estos últimos y R. Dworkin ${ }^{42}$

La propuesta de Nino respecto del problema de la relevancia del Derecho es anticipatoria de uno de los ejes del debate entre el positivismo excluyente (ELP) y el positivismo incluyente (ILP), ${ }^{43}$ y entre estas dos posiciones y Ronald Dworkin.

Tanto una como otra variante del positivismo coincidieron, siguiendo a Hart, en que las normas jurídicas tienen una textura abierta y que por ello (es decir, porque el lenguaje en el que se expresan las normas jurídicas no es unívoco sino que posee múltiples referencias posibles e incluso contradictorias, dando lugar a muchas proposiciones nor-

42 En la elaboración de este epígrafe debo mucho a las ideas de Pilar Zambrano. Cfr., al respecto, Zambrano, Pilar, La inevitable creación en la interpretación jurídica. Una aproximación a la tesis de la discrecionalidad, México, UNAM, 2009, pp. 7-36.

43 Cfr. Etcheverry, Juan B., El debate sobre el positivismo juridico incluyente. Un estado de la cuestión, México, UNAM, 2006, passim. 


\section{LA CONEXIÓN ENTRE DERECHO Y POLÍTICA}

mativas lógicamente correctas) resulta inevitable que su interpretación se conecte, en algún punto, con valores o principios morales. ${ }^{44} \mathrm{El}$ problema que aparece es en este punto es el siguiente: ¿cómo evitar, entonces, la confusión entre Derecho y Moral? ¿Cómo evitar que la apertura del lenguaje jurídico al lenguaje moral no acabe en la confusión de ambos niveles o "juegos de lenguaje"? ¿Cómo garantizar, en otras palabras, que el Derecho haga alguna diferencia en el razonamiento práctico, una vez que se constata que los enunciados normativos jurídicos reenvian a enunciados normativos morales?

Una de las respuestas más elaboradas a estas preguntas es la que propuso Joseph Raz (desde el ELP), quien sugirió distinguir de modo tajante entre el razonamiento sobre el Derecho y el razonamiento de acuerdo al Derecho. ${ }^{45} \mathrm{De}$ modo paralelo, Wilfred Waluchow (desde el ILP) propuso separar la identificación de la interpretación del Derecho. ${ }^{46} \mathrm{Se}-$ gún se sigue de los trabajos de ambos autores, el juicio acerca de la existencia de una norma jurídica (razonamiento sobre el derecho, o identificación del Derecho) es independiente del juicio interpretativo del Derecho (razonamiento de acuerdo a Derecho). El problema de la relevancia del Derecho se plantearía en el nivel del razonamiento sobre el Derecho o en el de su identificación, pero no en el del razonamiento de acuerdo al Derecho o en el de la interpreta-

44 Hart, Herbert L., The Concept of Law, 2a. ed. Oxford, Clarendon Press, p. 127.

45 Cfr. Raz, Joseph, Razón práctica y normas, trad. de J. Ruiz Manero, Madrid, Centro de Estudios Constitucionales, 1991, pp. 158-161. Del mismo autor, Between Authority and Interpretation. On the Theory of Law and Practical Reason, Oxford, Oxford University Press, 2009, esp. cap. III, pp. 223-370 y pp. 111-122. Sobre este autor, entre múltiples referencias, cfr. una descripción y una crítica sugerente en Seone, José Antonio y Rivas, Pedro, El último eslabón del positivismo jurídico. Dos estudios sobre Joseph Raz, Granada, Comares, 2005.

46 Waluchow, Wilfrid J., "In Pursuit of Pragmatic Legal Theory: the Practice of Principle by Jules Coleman" Canadian Journal of Law and Jurisprudence, Canada, vol. 15, 2002, pp. 141-150. 
ción. Es decir, lo realmente crucial para afirmar que el Derecho es relevante y hace una diferencia práctica consistiria en identificarlo (razonamiento sobre el Derecho) con prescindencia de consideraciones morales que resultarian inevitables en la interpretación (razonamiento de acuerdo al Derecho). El primero sería entonces el ámbito preciso en el que tendría sentido afirmar la desconexión entre Derecho y Moral.

Circunscripta así la tesis de la desconexión, desde el ELP no habria dificultad para luego afirmar que las indeterminaciones semánticas de los textos se deben resolver mediante el recurso a zonas de "no exclusión" de la Moral, ${ }^{47}$ como propone el propio Raz. Desde la perspectiva del ILP, en cambio, las indeterminaciones se resolverian acudiendo a "contenidos morales" que el Derecho mismo "incorporaria" al Derecho, como propone Waluchow. 48 En el primer caso, la tesis de la desconexión entre Derecho y Moral equivale a separación a la hora de la identificación; en el segundo, a separabilidad —es decir, a desconexión o conexión contingentes, y a desconexibilidad analítica-.

Esta última coincidencia fundamental entre el ELP y el ILP - es decir, la coincidencia en la desconexión (separación o separabilidad) entre Derecho y Moral - los hace pasibles de la misma crítica central: si bien se entiende cuál es la finalidad que se persigue al distinguir entre identificación e interpretación, por un lado, y entre conexión y separabilidad, por otro, —a saber: preservar la relevancia del Derecho-, lo cierto es que hay en ambas posiciones, por decirlo de algún modo, un error de objeto. El Derecho no es un conjunto de reglas como las del ajedrez — ni todo el Derecho ni una parte de él-, sino una práctica interpretativa, tal

47 Raz, Joseph, "Incorporation by Law", Legal Theory, USA, vol. 10, 2004, p. 12.

48 Cfr. Waluchow, Wilfrid J., Inclusive Legal Positivism, Oxford, Clarendon Press, 2003, pp. 240 y ss. 
como sostiene Dworkin, ${ }^{49}$ y esa indole hace impractible separar por completo la identificación de la interpretación. Sólo a través de la interpretación es posible llegar a la identificación: no es analíticamente posible identificar a una práctica interpretativa a la que no se ha interpretado. Identificar al Derecho es identificar una praxis sucesiva de interpretaciones, y consiste por tanto en ingresar en el horizonte de comprensión, de recreación del espíritu, del resto de los participantes de la práctica - de modo inmediato lo más frecuente es que se trate del autor de una norma o de una sentencia cuya relevancia para el caso se está analizando-.

Tomemos, para exponer esto último de modo más claro, un ejemplo de la jurisprudencia comparada. En el caso "Massa, J. A., c/ Poder Ejecutivo Nacional",50 la Corte Suprema argentina se propuso decidir "de modo definitivo" la cuestión "tan largamente debatida" (cons. 8) del llamado "corralito". 51 Mediante este último se impusieron dos res-

49 Cfr. Dworkin, Ronald, Law's Empire, Cambridge, Harvard University Press, 1986, pp. 225-227.

50 Cfr. Fallos (Colección Oficial de Fallos de la Corte Suprema de Justicia de la Nación), 329:5913, 2006.

51 Se trata del nombre con el que se conoció en Argentina a un conjunto de normas de emergencia instauradas entre fines de 2001 y comienzos de 2002. La primera de ellas fue el decreto 1570/01, que estableció, en su artículo 2, inciso a), la prohibición de "los retiros en efectivo que superen los pesos doscientos cincuenta $(\$ 250)$ o dólares estadounidenses doscientos cincuenta (US\$250), por semana, por parte del titular, o de los titulares que actúen en forma conjunta o indistinta, del total de sus cuentas en cada entidad financiera". Los depósitos se encontraron a partir de entonces, en el decir popular, "acorralados". A esto le siguió la pesificación, es decir, la conversión de las deudas en dólares en deudas en pesos, según un patrón de cambio distinto del valor de mercado que por entonces tenía la moneda estadounidense. Cfr. al respecto, entre abundante bibliografia, Ibarlucía, Emilio, "La emergencia económica y la interpretación constitucional”, en Cianciardo, Juan (coord.), La interpretación en la era del Neoconstitucionalismo. Una aproximación interdisciplinaria, Buenos Aires, Ábaco de Rodolfo Depalma, 2006, pp. 209-220. 
tricciones sobre los depósitos existentes en el sistema bancario argentino al momento de su entrada en vigencia. De una parte, se reprogramaron los plazos de vencimiento de los depósitos en forma indeclinable y, de otra, se dispuso la conversión de los depósitos en moneda extranjera a pesos, a una paridad "sensiblemente inferior a la del mercado libre de cambios" (cons. 2). Con el propósito de amortiguar el menoscabo producido por la conversión a pesos sobre los montos depositados, el artículo 4 del decreto 214/02 estableció que las sumas de dinero depositadas en moneda extranjera y convertidas a pesos se actualizarian de acuerdo con el llamado Coeficiente de Estabilización de Referencia (CER), atado a la evolución de la inflación, más la tasa de interés mínima fijada por el Banco Central para los depósitos, hasta el plazo en que venciese la reprogramación. Puesto que el plazo legal de la reprogramación del depósito de "Massa" ya había vencido al momento en que el caso llegó a conocimiento de la Corte, la cuestión que estrictamente tenía que resolver el Tribunal era la siguiente: ¿fue inconstitucional la conversión a pesos de la suma depositada? En palabras del propio Tribunal, se trataba de decidir si esta conversión dispuesta por el artículo 2 del decreto $214 / 02$ y sus normas complementarias y modificatorias había provocado "una mutación injustificada en la sustancia o esencia del derecho de los ahorristas" (cons. $2^{\circ}$ ). La Corte respondió negativamente a la pregunta planteada. Concluyó, en definitiva, que -en el caso concreto- la mutación del derecho de propiedad provocada por la normativa cuestionada no alteraba su esencia o sustancia. Para llegar a esta conclusión esbozó un razonamiento interpretativo analógico del artículo 4 del decreto 214/02.52

52 Vale la pena transcribir el texto de los primeros artículos del decreto mencionado:

"Artículo 1o.- A partir de la fecha del presente Decreto quedan transformadas a PESOs todas las obligaciones de dar sumas de dinero, de cualquier causa u origen - judiciales o extrajudiciales- expresadas EN DÓLARES ESTADOUNIDENSES, u otras monedas extranjeras, existentes a la 


\section{LA CONEXIÓN ENTRE DERECHO Y POLÍTICA}

Pues bien, la discusión en el caso giró en torno a la siguiente pregunta: ¿cuál es el contenido del derecho de propiedad? Yendo más a fondo: los operadores jurídicos argentinos que litigaron en "Massa" identificaron sin dificultad mayor la existencia de un "enunciado lingüístico" que protege la propiedad (el artículo 17 de la Constitución de ese país), pero unos y otros difirieron en la proposición normativa a la cual ese enunciado remite. Y la norma jurídica no es el enunciado, sino la proposición. 53

sanción de la Ley $\mathrm{N}^{\circ} 25.561$ y que no se encontrasen ya convertidas a PESOS.

Art. 2o.- Todos los depósitos en DÓlARES ESTADOUNIDENSES u otras monedas extranjeras existentes en el sistema financiero, serán convertidos a PESOS a razón de PESOS UNO CON CUARENTA CENTAVOS $(\$ 1,40)$ por cada DÓLAR ESTADOUNIDENSE, o su equivalente en otra moneda extranjera. La entidad financiera cumplirá con su obligación devolviendo PESOS a la relación indicada.

Art. 3o.- Todas las deudas en DÓLARES ESTADOUNIDENSES u otras monedas extranjeras con el sistema financiero, cualquiera fuere su monto o naturaleza, serán convertidas a PESOS a razón de UN PESO por cada DÓLAR ESTADOUNIDENSE o su equivalente en otra moneda extranjera. El deudor cumplirá con su obligación devolviendo PEsos a la relación indicada.

Art. 4o.- A los depósitos y a las deudas referidos, respectivamente, en los artículos 2o., 3o., 8o. y 11 del presente Decreto, se les aplicará un Coeficiente de Estabilización de Referencia, el que será publicado por el BANCO CENTRAL DE LA REPÚBLICA ARGENTINA. Además se aplicará una tasa de interés mínima para los depósitos y máxima para los préstamos. E1 coeficiente antes referido se aplicará a partir de la fecha del dictado del presente decreto".

53 Cfr. al respecto Finnis, John, "Helping Enact Unjust Laws without Complicity in Injustice", The American Journal of Jurisprudence, USA, vol. 49, 2004, p. 27. Sobre la sistematicidad como una cualidad fenomenológica y por lo mismo necesaria de toda interpretación jurídica cfr. Zambrano, Pilar, La inevitable creatividad en la interpretación jurídica (...), op. cit., pp. 65-73; "Los derechos ius-fundamentales como alternativa a la violencia: entre una teoría lingüística objetiva y una teoría objetiva de la justicia", Persona y Derecho, España, vol. 60, 2009, pp. 131-152; "E1 Derecho como práctica y como discurso. La perspectiva de la persona como garantía de objetividad y razonabilidad en la interpretación", Díkaion, Colombia, vol. 23, núm. 18, diciembre de 2009, pp. 109-133; "La interpretación jurídica 


\section{JUAN CIANCIARDO}

Lo anterior permite ver que la tesis de la desconexión es sustancialmente falsa. Paralelamente, vale la pena resaltar que la afirmación de la tesis de la conexión entre el Derecho y la Moralno se opone a la afirmación de su distinción. Sí se opone a la separación (ELP), a la separabilidad (ILP) y a la confusión (Cathrein). Esto es negado, claro, desde el pensamiento disyuntivista de la postmodernidad, que desconoce la analogía. ${ }^{54}$

\section{Contexto y critica de la propuesta de Nino}

La propuesta de Nino se relaciona, como es obvio, con el modo en el que se plantea la pregunta acerca de la conexión y de la relevancia. Y esto último se enmarca, a su vez, en una Ética (el constructivismo) y una Filosofia politica (la democracia deliberativa) muy concretas.

En mi opinión, esta contextualización resulta imprescindible para comprender a fondo esa propuesta, y para entender, asimismo, cuáles son sus límites y condicionamientos centrales. Como sostuvo Pilar Zambrano respecto de Dworkin, Nino es un ejemplo de quienes tienden "a tomar una postura crítica respecto del "positivismo" sin asumir en toda su extensión los presupuestos epistemológicos en que se asienta esa crítica". ${ }^{55}$ Veamos.

como co-creación", Problema. Problema. Anuario de Filosofía y Teoría del Derecho, núm. 3, 2009, pp. 375-414.

54 Cfr. Ballesteros, Jesús, Postmodernidad: decadencia o resistencia, Madrid, Tecnos, 1989, p. 22. Sobre la analogía y el disyuntivismo, cfr., asimismo, Llano, Alejandro, La nueva sensibilidad, Madrid, Espasa-Calpe, 1988, pp. 212-233; y Kaufmann, Arthur, Analogía y naturaleza de la cosa: hacia una teoría de la comprensión jurídica, Santiago de Chile, Editorial Jurídica de Chile, 1976, passim.

55 Zambrano, Pilar, "Objetividad en la interpretación judicial y objetividad en la moral (...)”, op. cit., pp. 281-326 y 283. En el caso de Nino aparece, además, la pretensión de contribuir desde la Teoría del Derecho a evitar violaciones a los derechos humanos como las que se vivieron en Argentina durante el último gobierno militar (1976-1983), cuyo impacto 


\section{LA CONEXIÓN ENTRE DERECHO Y POLÍTICA}

(a) Dialéctica de la Modernidad, constructivismo ontológico y constructivismo epistémico

Una crítica global proviene precisamente de la Ética y la Filosofia política liberales que postula Nino. Se trata de una paradoja inevitable a la que conduce la "dialéctica de la Modernidad": 56 en aras de fomentar la libertad, el planteamiento de nuestro autor acaba dejando prácticamente sin margen de actuación a quien esté en desacuerdo con la mayoría. Continuando con la analogía que el propio Nino nos propone, no hay espacio para aquellos músicos cuyos criterios estéticos no coincidan con el de la orquesta.

La fundamentación de esto último exige avanzar algo más, abriendo las puertas a una segunda crítica complementaria. Nino se propuso elaborar una teoría moral que permita la superación del dogmatismo y del escepticismo sin renunciar a la objetividad ética. ${ }^{57}$ Ideó, para ello, una

en el trabajo académico de este autor es evidente. Cfr., p. ej., su "Introduction" a Nino, Carlos (ed.), Rights, Darthmouth, Aldershot, 1992, XI-XXXIV.

56 Un análisis de esta expresión y de sus implicancias en Innerarity, Daniel, Dialéctica de la modernidad, Madrid, Rialp, 1990. Cfr., asimismo, Alvira, Rafael, "Dialéctica de la modernidad", DADUN, EBSCOhost, viewed 11 January 2012.

57 Nino es uno entre los muchos autores movidos por una preocupación que Massini identifica como clave para la iusfilosofía contemporánea: "la búsqueda de una cierta instancia de objetividad ético-jurídica, pero sin que sea necesario recurrir a una concepción cognitivista, y por lo tanto veritativa, de la eticidad. Dicho de otro modo, de lo que se trata para estos autores es de alcanzar las ventajas innegables del iusnaturalismo clásico: su presentación de un fundamento fuerte de la normatividad jurídica y su aporte de un criterio objetivo de estimación ética, sin comprometerse con la existencia de normas de carácter inexcepcionable, ni con la necesidad de descubrir en la realidad los contenidos de los bienes humanos básicos, con la consiguiente adopción de una postura cognitivista respecto de las realidades éticas y en especial de las jurídicas. La gran mayoría de las corrientes enroladas en esta tercera alternativa entre iusnaturalismo y iuspositivismo adoptan, explícita o implicitamente, una concepción constructivista de la normatividad ética, es decir, una visión 
teoría del discurso moral que lo condujo en una primera etapa a un constructivismo ontológico que, luego, en una etapa posterior, rechazó y cambió por un constructivismo epistemológico. Desde su perspectiva, el gobierno, la democracia y las normas son los vehículos efectivos en el que tal discurso moral se desarrolla, aunque tienen, insisto, valor epistémico, y no ontológico. ${ }^{58}$

Desde la perspectiva que surge del análisis de la paradoja de la irrelevancia se percibe, en mi opinión, que las consecuencias prácticas de esta última distinción acaban siendo irrelevantes, lo que conduce al autor a una inconsistencia importante. Veamos porqué. Según Nino, la democracia no se identifica con el discurso moral (si esto ocurriese, estaríamos ante un constructivismo ontológico) pero es su sucedáneo. Por eso existe la posibilidad de que las normas producidas por la democracia no sean moralmente válidas. Dicho de otro modo: las normas que surgen de una democracia nos permiten conocer las normas morales, puesto que el discurso democrático funciona como sucedáneo del discurso moral, pero, sin embargo, no todas las normas democráticas son morales, porque de otro modo no habría límites a la actuación de las mayorias y la democracia no podría fundamentar los derechos (constructivismo ontológico). Aquí nos topamos con dos problemas: el de explicar cómo

según la cual los principios ético-jurídicos son de algún modo construidos o inventados o elaborados por los hombres a través de algún procedimiento establecido de la racionalidad práctica. Dicho en otras palabras, la razón práctica, desprovista de todo supuesto contenutístico dado objetivamente, establece sus puntos de partida y las reglas de su procedimiento inferencial, arribando a principios éticos que no son la derivación práctica de un conocimiento de la realidad, sino el resultado de una mera construcción mental-social" (Massini, Carlos, Constructivismo ético y justicia procedimental en John Rawls, México, UNAM, 2004, pp. 8-9).

58 Cfr. una explicación más extensa de estas ideas, con las correspondientes citas, en Cianciardo, Juan, "La paradoja de la irrelevancia moral del gobierno y del Derecho. Una aproximación desde el pensamiento de Carlos Nino", Díkaion. Revista de fundamentación jurídica, Colombia, vol. 19, núm. 1, junio de 2010, pp. 47-70 y 59-68. 
es posible conocer si las normas democráticas son o no válidas, siendo la democracia el sucedáneo del discurso moral, por un lado, y el de establecer qué debe hacerse, en su caso, frente a la invalidez moral de esas normas, por otro.

El primer problema es muy dificil de resolver para Nino, y su propuesta acaba en la conclusión de que, "salvo error, en los países democráticos es probable que sea obligatorio obrar conforme a Derecho". 59 Con respecto a lo segundo, y como consecuencia de lo anterior, como señala Blanco, "Nino exige que, a efectos prácticos, se tome esa probabilidad como certeza a la hora de actuar. Apelando al principio de que se deben maximizar las posibilidades de obrar de forma moralmente correcta, Nino cree que el discurso democrático —incluso imperfecto- justifica y aun exige relegar el propio juicio y acatar la norma". 60

Nino define a las normas jurídicas como "razones epistémicas". Es decir, razones para creer que existen razones para actuar. Con esto busca preservar la autonomía de la moral (que se vería cuestionada, en su opinión, si existiesen autoridades prácticas), y, de modo simultáneo, huir del positivismo ideológico (en el que se incurriría si se definiese a las normas, sin más, como razones que desplazan todo otro tipo de razones) sin dejar de afirmar la obligatoriedad del derecho positivo. ${ }^{61} \mathrm{El}$ intento, sin embargo, se enfrenta a dificultades insalvables: si se entiende que para el constructivismo epistemológico las normas democráticas actúan como razones excluyentes (en el sentido de concluyentes), la autonomía, presupuesto del discurso moral, queda en entredicho, ya que los destinatarios de las normas jurídicas siempre habrán de relegar su juicio, adoptando la norma como única guía de actuación. "Si, por el contrario, se ad-

59 Blanco Miguélez, Susana, Positivismo metodológico y racionalidad política: una interpretación de la teoría jurídica de Carlos S. Nino, Granada, Comares, 2002, p. 151.

60 Ibidem, p. 152.

61 Ibidem, p. 153. 
mite con Nino que son razones epistémicas (...), cuando se afirma que un sujeto ha llegado por reflexión individual al convencimiento de que las normas democráticas presentan errores morales relevantes y a la vez se le exige que "crea en la existencia de razones morales a favor de esas normas", se le está pidiendo en realidad que acepte simultáneamente dos criterios de corrección, sin ofrecerle ningún argumento definitivo para primar uno sobre otro".62

En definitiva, como afirma Blanco, la propuesta de Nino acaba teniendo un cierto aire de familia con el positivismo ideológico, pese a sus declaraciones de intentar un resultado exactamente contrario. ${ }^{63}$ En efecto, de sus ideas se deriva que excepto en los casos de error es probable que sea obligatorio obrar conforme al Derecho, y que, no obstante ello, es exigible a efectos prácticos que esa posibilidad sea tomada como certeza a la hora de actuar. "Apelando al principio de que se deben maximizar las posibilidad de obrar de forma moralmente correcta, Nino cree que el discurso democrático -incluso imperfecto- justifica y aun exige relegar el propio juicio y acatar la norma". ${ }^{64}$ La búsqueda de Nino de garantizar la obediencia de este modo y con este alcance ha sido por eso definida, con razón, como "legalismo ético democrático". 65

\section{(b) Indeterminación y falta de conclusión}

Como se ha visto más arriba, Nino rechaza que la falta de determinación y conclusión de la Moral puedan proporcionar relevancia al Derecho. Sostiene en este sentido, con acierto, que no es posible resolver esas indeterminaciones y faltas de conclusión sin acudir de algún modo a la Moral.

62 Idem.

63 Ibidem, pp. 155-156.

64 Ibidem, p. 152.

65 Pozzolo, Susanna, "Note su Derecho, moral y política di Carlos S. Nino", en P. Comanducci y R. Guastini, Analisis e diritto 1997, Ricerche di giurisprudenzaanalitica, Giappichelli, Torino, 1998, pp. 119-144.

160 


\section{LA CONEXIÓN ENTRE DERECHO Y POLÍTICA}

Siendo esto así, la nueva (e inevitable) incursión en la Moral se enfrentaría otra vez a indeterminaciones y faltas de conclusión que re-conducirian al Derecho, incurriéndose así en un círculo vicioso. Esto le conduce a buscar en otro lugar la relevancia, que sitúa finalmente en el carácter político del fenómeno jurídico. Formularé a continuación, de modo sintético, tres críticas u objeciones que merece, en mi opinión, este planteamiento.

a) Debe tenerse presente que también la Moral es una práctica colectiva. El hombre no adopta decisiones morales prescindiendo por completo de los demás. En la propuesta de Nino subyace como trasfondo una Ética solipsista tipicamente liberal, contra la que se ha alzado la crítica comunitarista, ${ }^{66}$ entre otras. ${ }^{67}$ En primer lugar, porque sus decisiones morales involucran más o menos directamente — según los casos - a otros. En segundo lugar, porque su identidad es social, es decir, se enfrenta a los problemas y los resuelve desde una cultura con la que él mismo en buena medida se encuentra, y que le es proporcionada por otros seres humanos. Siendo esto así, no es posible distinguir al Derecho de la Moral afirmando que sólo el primero es colectivo o social.

b) Más allá de la crítica anterior, si se sigue el razonamiento expuesto más arriba, se constatará que según Nino el apuntado carácter político proporcionaria al Derecho algo que la Moral — partiendo del pluralismo existente- no tiene: capacidad de coordinar conductas y de resolver conflictos. Desde esta perspectiva, la relevancia del Derecho provendría en última instancia precisamente de aquello a lo que Nino le había negado ese efecto: la indeterminación y falta de conclusión de la Moral, incapaz de establecer por sí sola cómo debe procederse frente a las demandas politicas

66 MacIntyre, Alasdair, After Virtue: A Study In Moral Theory, 3a. ed., Londres, Duckworth, 2007; del mismo autor, Ethics And Politics, Cambridge, UK-New York, Cambridge University Press, 2006.

67 Cfr., p. ej., Cruz Prados, Alfredo, Ethos y polis. Bases para una reconstrucción de la filosofía política, Pamplona, Eunsa, 1999, passim. 
de coordinación y de resolución de conflictos. Dicho con otras palabras, el autor argentino acaba afirmando al final de su desarrollo teórico aquello que había negado al comienzo. Y no podía ocurrir de otro modo puesto que, en efecto, y aunque no me detendré en este punto, la relación entre Derecho y Moral es fundamentalmente una relación de determinación: el Derecho determina aquello que la Moral no determina. 68

c) Esta última crítica se relaciona, en mi opinión, con la perspectiva epistemológica desde la que Nino se plantea la pregunta acerca de la relevancia. Se trata de un punto de vista que parece desconocer el funcionamiento de la razón práctica. En el fondo, la determinatio no es sino un ejemplo craso de cómo opera la razón práctica y de qué es lo que alcanza (la verdad práctica), con el añadido de que en el ámbito del Derecho se mezcla además con la razón poiética (pues elabora productos como normas y sentencias). ${ }^{69}$ Esto es compatible, desde mi punto de vista, con la aceptación de que en todo razonamiento jurídico existe un envío y reenvío entre caso y norma, entre normas y principios, entre Derecho y Moral. No hay aquí, sin embargo, necesariamente un círculo vicioso, que es en todo caso una amenaza de la que se sale no de modo lineal, sino a través de un espiral: el círculo se disuelve si se advierte, por un lado, que las decisiones prudenciales que conducen a la determinación de la moral son un producto mixto de razón y voluntad y, por otro lado, que esas decisiones - precisamente por la dependencia simultánea entre razón y voluntad- son inteligibles y justificables.

68 Cfr., al respecto, Massini Correas, Carlos y García Huidobro, Joaquín, "Valoración e inclusión en el Derecho. La máxima "lex injusta non est lex" y la iusfilosofía contemporánea”, en Cianciardo, J. et al., Razón jurídica y razón moral. Estudios sobre la valoración ética en el Derecho, México, Porrúa, 2012, pp. 109-137.

69 Agradezco esta idea al profesor Pedro Rivas. Cfr., al respecto, Llano, Carlos, Examen filosófico del acto de la decisión, Pamplona, Eunsa, 2010, passim. 
Un intento como él lo propone, sólo resultará fructífero si se acepta la presencia de algún referente ontológico (de uno o varios bienes humanos, de la persona, en definitiva), que lo dote de sentido. ${ }^{70}$ No parece posible afirmar la tesis de la conexión con vigor, desde el escepticismo ético o desde una Ética diluida o difusa. Afirmar la conexión del Derecho con cualquier Ética, o con una Ética en la que todo cabe, equivale a negarla y conduce inevitablemente al nihilismo jurídico. Los principios, en definitiva, nos envian a un referente ontológico. Por otro lado, la indeterminación del Derecho nos envía a una teoría de la acción humana, que opera como su margen de referencia. ${ }^{71} \mathrm{La}$ inteligibilidad del intento dependerá, por último, de que tanto esa teoría de la justicia como la de la acción se conecten con una teoría semántica que permita ligarlas entre sí. ${ }^{72}$

70 Cfr. Zambrano, Pilar, La inevitable creación en la interpretación juridica (...), op. cit., passim.

71 Agradezco esta idea a la profesora Pilar Zambrano, desarrollada recientemente en "Principios, razones y derecho. Una aproximación al Derecho como diferencia práctica", [manuscrito].

72 Cfr., op. cit., Zambrano, Pilar, "El Derecho como práctica y como discurso. La perspectiva de la persona como garantía de objetividad y razonabilidad en la interpretación”, pp. 20-40. 
DR (C) 2012, Universidad Nacional Autónoma de México,

Instituto de Investigaciones Jurídicas 